\title{
DEVELOPMENT OF A SYSTEMIC EVOLUTIONARY AND BIOCYBERNETIC METHODOLOGY USING THE CHAOS AND CATASTROPHES THEORIES TO HANDLE COMPLEX DECISION SITUATIONS IN MANAGEIMIINT
}

Veneta Marzen, University of Southampton, UK

A critical review of many existing methodologies shows that these could not cope with the apparent complexity of the real world. In this sense, the limits of analytical approaches (e.g. MS / OR, MCDM) have been analyzed in relation to the recognition of the chaos theory which questioned the validity of the Newtonian deterministic paradigm. However complexity of a decision situation could not only be related to different states of an open system. Rather complexity is concerning with the whole human social system. In this sense complexity involves the degree of inter connectivity of all hierarchical levels in the decision process, their cross-over links, feedbacks and also consequences for the decision field.

The phases of the proposed decision-aid/planning methodology for complex decision situations are summarised as follows:

Phase 1: Take a block of system's variables covering all aspects of our life. Reduce the complexity of the system by taking only relevant $k \in \mathrm{y}$ factors able to create a rough picture of its reality.

Phase 2: Find out the inter dependencies of all relevant systemic variables.

Phase 3: Determine the active, passive, critical and buffer elements.

Phase 4: Elaborate time aspects. Follow future development paths and simulate if possible all scenarios in the form of systems dynamic or causal-loops diagrams.

Phase 5: Build a controlling model and show the controllable and noncontrollable elements. Build a policy test.

phase 6: Use the biocybernetic checklist. Determine controlling actions based on cybernetic and biocybernetic rules.

These methodology was used to analyze the EC project MARTA which aims to introduce telematics for control of future traffic flows in Europe. 